\title{
8
}
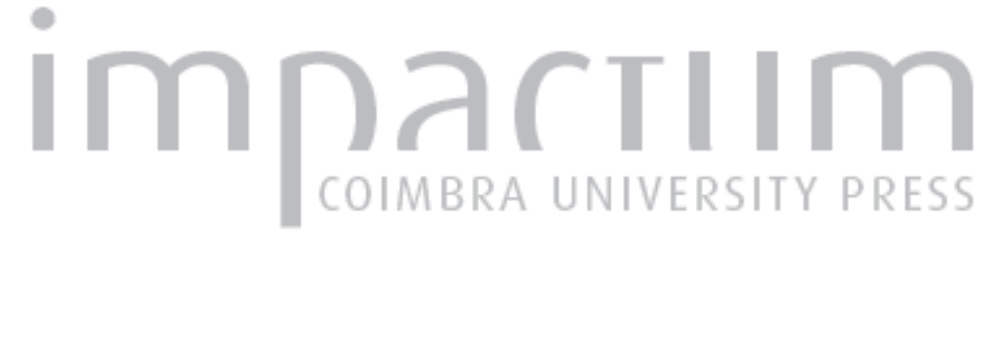

\section{Colisión por alcance posterior y lesión infrecuente: absceso subclavicular y axilar por estreptococo pyogenes} \author{
Autor(es): $\quad \begin{aligned} & \text { Hernando Lorenzo, AE; Aguado de los Reyes, J; Ortega, Al; Gómez- } \\ & \text { Guillamón, FGN }\end{aligned}$
}

Publicado por: Imprensa da Universidade de Coimbra

URL

persistente:

URI:http://hdl.handle.net/10316.2/43561

DOI:

DOI:https://doi.org/10.14195/1647-8630_27_5

Accessed : $\quad$ 26-Apr-2023 14:45:38

A navegação consulta e descarregamento dos títulos inseridos nas Bibliotecas Digitais UC Digitalis, UC Pombalina e UC Impactum, pressupõem a aceitação plena e sem reservas dos Termos e Condições de Uso destas Bibliotecas Digitais, disponíveis em https://digitalis.uc.pt/pt-pt/termos.

Conforme exposto nos referidos Termos e Condições de Uso, o descarregamento de títulos de acesso restrito requer uma licença válida de autorização devendo o utilizador aceder ao(s) documento(s) a partir de um endereço de IP da instituição detentora da supramencionada licença.

Ao utilizador é apenas permitido o descarregamento para uso pessoal, pelo que o emprego do(s) título(s) descarregado(s) para outro fim, designadamente comercial, carece de autorização do respetivo autor ou editor da obra.

Na medida em que todas as obras da UC Digitalis se encontram protegidas pelo Código do Direito de Autor e Direitos Conexos e demais legislação aplicável, toda a cópia, parcial ou total, deste documento, nos casos em que é legalmente admitida, deverá conter ou fazer-se acompanhar por este aviso.

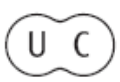


ISSN: 1645-0760

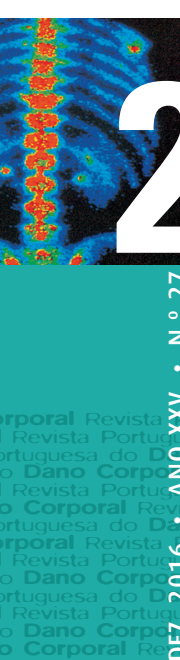

REVISTA PORTU/GUESA
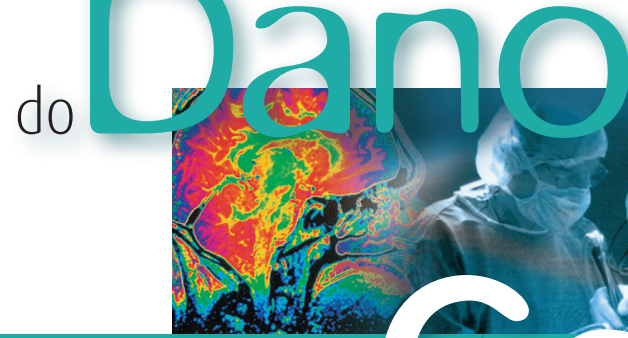

N

z.

希ort

Do

: inta Cortuguesa do Dano Corporal Revista Portuguesa do Dano Corporal Revista Portuguesa

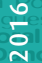

ầं।

Corporal Revista Portuguesa do Dano Corporal Revista Portuquesa do Dano Corporal

I Revista Portuguesa do Dano Cortuguesa do Dano Corporal Revista Portugues
Revista Pdrtuguesa do Dano Corporal

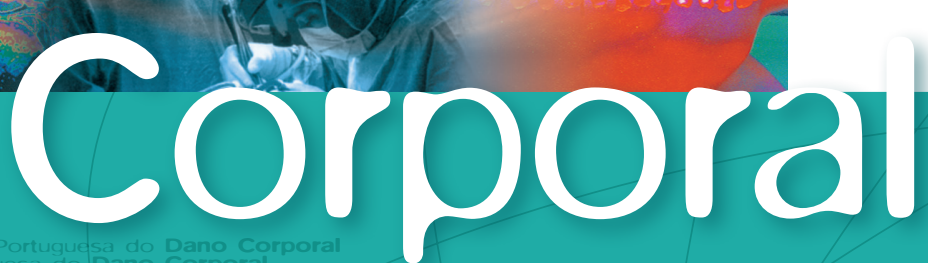

APADAC

ASSOCIAÇÃO PORTUGUESA

DE AVALIAÇÃO

DO DANO CORPORAL 


\section{Colisión por alcance posterior y lesión infrecuente: absceso subclavicular y axilar por estreptococo pyogenes}

Hernando Lorenzo AE ${ }^{1}$, Aguado de los Reyes $\mathrm{J}^{2}$, Ortega $\mathrm{AI}^{3}$, Gómez-Guillamón FGN ${ }^{4}$

Resumo: Colisão posterior e lesão rara: abcesso subclavicular e axilar por estreptococo pyogenes.

Reporta-se um caso de abcesso subclavicular e axilar por estreptococo pyogenes, decorridos alguns dias depois de uma colisão posterior, numa condutora com lesões dos tecidos moles cervicais e luxação acromioclavicular; comenta-se a raridade desta entidade e discute-se o nexo de causalidade.

Palavras-chave: Colisão posterior; lesão rara; abcesso subclavicular e axilar; estreptococo pyogenes.

Summary: Rear-end collision and rare injury: subclavicular and axillary abscess by streptococcus pyogenes.

A case of subclavicular and axillary abscess by streptococcus pyogenes, in a female driver, some days after a rear-end low speed collision is reported. The driver suffered "whiplash injury" and an acromioclavicular luxation; this rare entity and the medico-legal relationship accident-injury is discussed.

Key-words: Rear-end collision; rare injury; subclavicular and axillary abscess; streptococcus pyogenes.

1 Médico. Especialista en Medicina Intensiva y Cardiología. Magister Universitario en VDC.

2 Médico. Especialista en Medicina Interna. Magister Universitario en VDC.

3 Médico. Especialista en Medicina Interna. Magister Universitario en VDC.

4 Médico. Especialista en Medicina Intensiva y Anestesiología Y Reanimación. Magister Universitario en VDC. 


\section{DESCRIPCIÓN DEL CASO}

Se produjo un accidente de circulación en ámbito urbano, consistente en colisión por alcance posterior de un turismo Dacia Lodgy matrículado en 2013, que colisionó con su parte anterior contra la parte trasera de un turismo Fiat Brava matriculado en el año 2000.
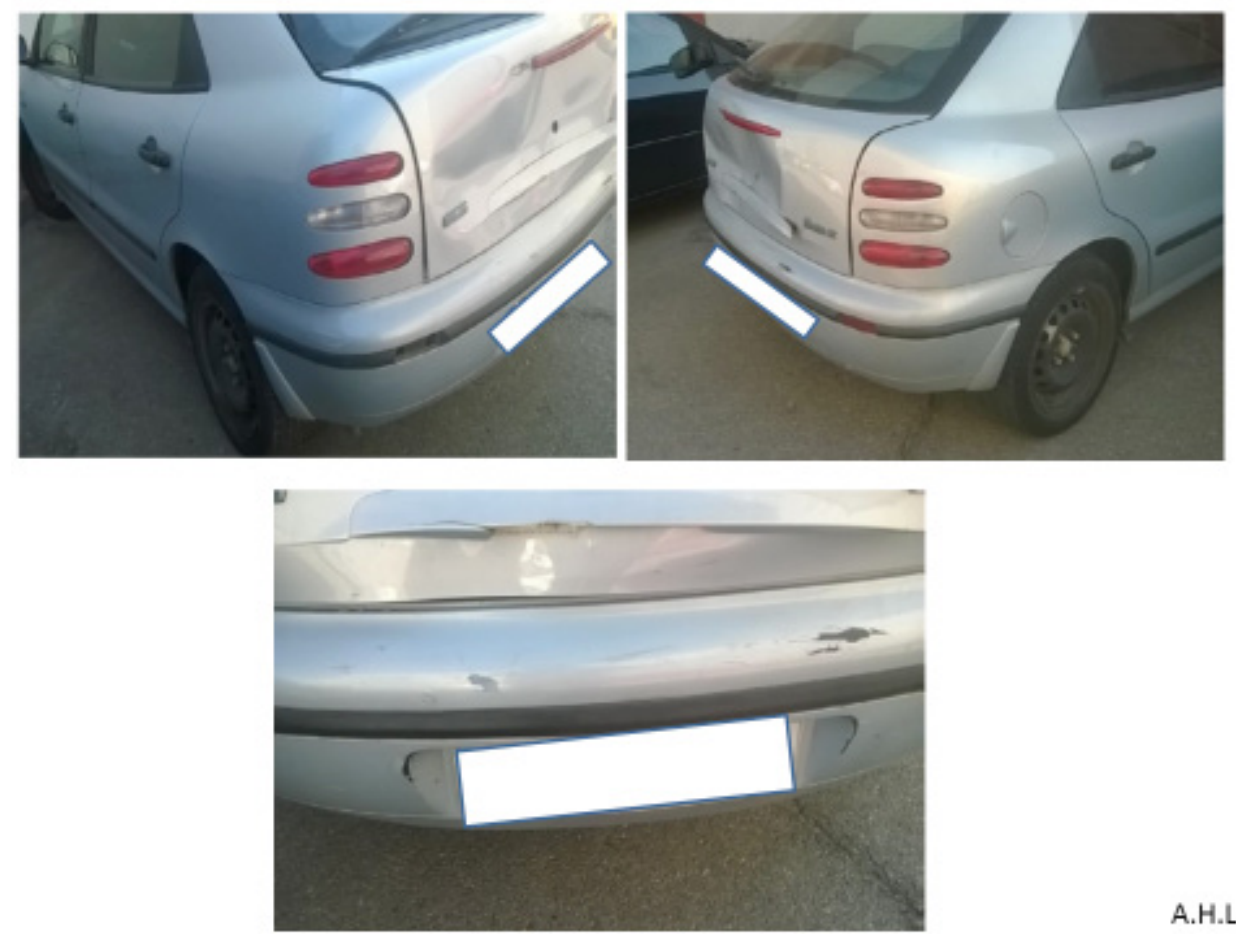

A.H.L.

Fig 1.

Los costes de reparación del turismo Fiat Brava, que afectaban a portón trasero, parachoques, eran de 744 Euros, (IVA incluído)

Los daños en el vehículo dacia Lodgy, afectaban a la parte delantera, (parachoques, capó, rejilla, ambos faros, radiador, con un coste de reparación de 1352 Euros (IVA incluído)

Como consecuencia del accidente, la conductora del turismo Fiat Brava, mujer de 45 años de edad, fue vista en Urgencias del Hospital. Refería dolor en esternocleidomastoideo y trapecio izquierdo sin irradiación, teniendo el 
balance articular cervical limitado por dolor. Fue diagnosticada de esguince cervical.

Quince días después del accidente, es vista en otro hospital por dolor en hombro derecho y parrilla costal derecha, refiriéndose los diagnósticos de omalgia derecha, tendinitis bicipital, esguince cérvico-dorsal, lumbalgia y coxigodinia tras accidente de tráfico.

Una semana después acude al hospital por dolor intenso en hombro, con imposibilidad para mover el hombro y dolor a la palpación en articulación acromioclavicular, siendo diagnosticada tras $\mathrm{Rx}$ de hombro, de luxación acromioclavicular grado II, siendo inmovilizada en cabestrillo.

Cinco días después es vista en Urgencias del Hospital Universitario por fiebre de $39,5^{\circ}$ desde cuatro días antes, tratada con ciprofloxacino, con empeoramiento inicial de los síntomas; consulta por poliartralgias y aporta analítica de una semana antes, con hemograma y bioquímica normales.

A la exploración se refería miembros inferiores con leves edemas maleolares bilaterales sin otros signos inflamatorios. Fue diagnosticada de poliartrialgias inespecíficas sin signos de alarma y cistitis no complicada prescribiéndose tratamiento con fosfomicina, paracetamol e hidratación abundante. Dos días después ingresó en el hospital, tras acudir a Urgencias al persistir la fiebre, observándose hiperbilirrubinemia y elevación leve de transaminasas.

Dos semanas después es dada de alta del Hospital Universitario, refiriéndose que presentó artralgias generalizadas con eritema articular e hinchazón y dolor en el tendones, debilidad que le impide la movilización, edemas con fóvea en miembros inferiores, exantema que se resuelve con descamación y orinas de color oscuro. Al ingresar en Servicio de Aparato Digestivo, presentaba fiebre de 39, TA de 104/63, frecuencia cardiaca de 100 lpm y discreta ictericia mucocutánea. Las articulaciones de manos estaban dolorosas y calientes sin signos visuales inflamatorios.

El hombro derecho doloroso, con inflamación que se extiende a cuello y tórax, presentando impotencia funcional, siendo incapaz de levantarlo a más de $30^{\circ}$. Se palpa además bulto rojizo y caliente en zona anterior de línea axilar y se aprecia miembro superior derecho hinchado y con aumento del grosor de vías periféricas. En analítica destacaba 19.650 leucocitos con una bilirrubina de 3,29.

En TAC de hombro se observaba imagen de absceso de partes blandas, con gran colección de contornos anfractuosos que diseca desde región infraclavicular derecha en sentido caudal, englobando parcialmente plexo braquial, extendiéndose hacia musculatura pectoral y región axilar con pequeñas adenopatías reactivas y delimitado posteriormente por el músculo subescapular. 
Los hallazgos radiológicos eran compatibles con absceso de partes blandas, que se extiende desde región infraclavicular derecha hacia región axilar y anterolateral de pared torácica, realizándose punción en la zona flemonosa, apareciendo pus amarillento, que tras estudio por Microbiología mostró estreptococo pyogenes.

Un TAC torácico confirma gran absceso en zona de hombro y se inicia antibioterapia con cefazolina, procediéndose el 27 de enero a drenaje quirúrgico de la cavidad por Servicio de Cirugía de Tórax, con abundante salida de pus, resección de esfacelos dejando drenaje que se retira a la semana.

El diagnóstico fue hematoma abscesificado tras traumatismo (accidente de tráfico en hombro derecho) y celulitis por estreptococus pyogenes, que debuta como sepsis grave.

\section{DISCUSION Y COMENTARIOS}

En síntesis, se trata de una paciente de 45 años, conductora de un turismo, que sufre colisión por alcance posterior, siendo diagnosticada inicialmente de esguince cervical.

A los pocos días, refiere dolor y presenta limitación funcional en hombro derecho, siendo diagnosticada de luxación acromioclavicular (se refiere en un informe grado I y en otro informe grado II).

La articulación acromioclavicular está frecuentemente afectada en las lesiones traumáticas que se localizan en el hombro. Las lesiones a la articulación suponen aproximadamente el $12 \%$ de las que se ven en la articulación del hombro en la práctica clínica, aunque es probable que esta cifra esté subestimada respecto a la prevalencia verdadera, ya que pacientes con lesiones leves pueden no buscar asistencia médica.

La causa más frecuente de la lesión en la articulación acromioclavicular es una fuerza aplicada directamente sobre el borde superolateral del hombro, generalmente durante una caída con el hombro en addución. Esta fuerza lleva a la clavícula y el acromion inferiormente con los fuertes ligamentos de unión de la articulación acromioclavicular limitando la cuantía de desplazamiento inferior de la clavícula. El mayor grado de la trasposición inferior del acromion puede entonces llevar a la rotura de la acromioclavicular y los ligamentos coracoclaviculares.

Rockwood S. y su grupo (Rockwood CA, Williams GR, Young DC., Acromioclavicular injuries. In: Rockwood CA, Green DP, Bucholz RW, Heckman JD, editors. Fractures in Adults. 4th ed. Vol I. Philadelphia, PA: Lippincott-Raven; 1996, 1341-1413) desarrollaron la clasificación más 
ampliamente aceptada para las lesiones de la articulación acromioclavicular, basados en el trabajo original de Tossy y cols. de 1963 (Tossy JD, mead NC y cols., Acromioclavicular separations: useful and practical classification for treatment., Clin. Orthop. Relat. Res. 1963; 28:111-19), y en el de Allman, 1967 (Allman FL. Fractures and ligamentous injuries of the clavicle and its articulation. J Bone Joint Surg Am. 1967;49 (4): 774-84. J Bone Joint Surg Am).

La clasificación incluye seis tipos de lesión a la articulación acromioclavicular que se basan en la gravedad de la lesión sufrida por los ligamentos acromioclaviculares y los ligamentos coracoclaviculares además de los tejidos de soporte que incluyen los músculos deltoides y trapecio.

Las lesiones menos graves de la clasificación de Rockwood (tipo I-III) generalmente se tratan conservadoramente mientras que el tipo IV y los siguientes a menudo precisan tratamiento quirúrgico.

La luxación acromioclavicular suele clasificarse como de grado I, II y III, de acuerdo a la separación existente entre el acromion y la clavícula.

Respecto a la clasificación, en 1963 Sege y Salvatore proponen una clasificación de la luxación acromioclavicular en tres tipos que son semejantes a la de Rockwood, quien a su vez se había apoyado en la clasificación de Tossy y cols., de 1963.

Rockwood CA, (Injuries to the acromioclavicular joint. In:Rockwood CA, Green DP eds., Fractures in adults, Vol. 1, 2nd edn. Philadelphia: JB Lippincott, 1984:860), clasificó en 4 tipos las lesiones acromioclaviculares, agregándole dos más posteriormente.

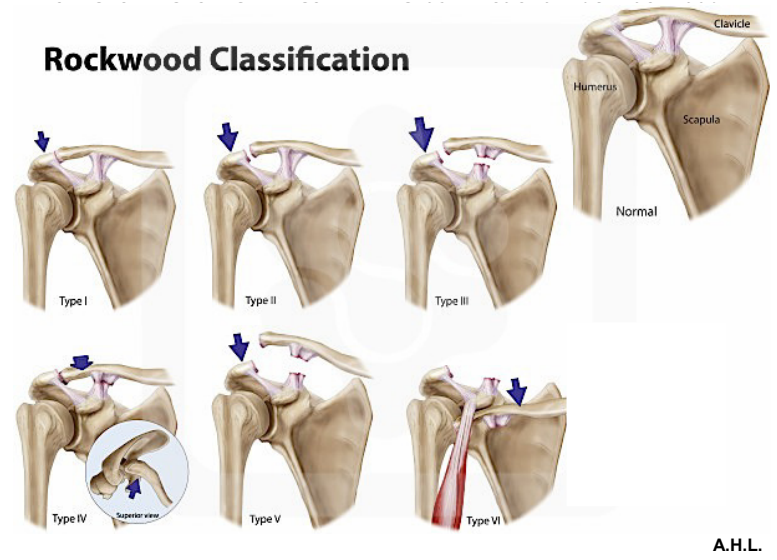

Fig 2. Luxación acromioclavicular - Clasificación de Rockwood 
TIPO 1. Cuando hay un accidente no trascendental en el hombro, no hay deformación del mismo, sólo dolor. Los ligamentos acromioclaviculares están normales y solamente después de dos semanas al tomarle una RX se aprecia que hay una pequeña calcificación en la zona acromioclavicular.

TIPO 2. Cuando hay ruptura de ligamentos acromioclaviculares y de la cápsula, hay ligera deformación del hombro, mucho dolor. La clavícula está inestable.

TIPO 3. Cuando el accidente es más violento y hay rotura del ligamento esternoclavicular, cápsula, ligamento coracoclavicular y desinserción del deltoide en la zona clavicular, se produce una gran separación de la articulación por la tracción del trapecio. Estas tres son semejantes a la clasificación de Pege y Salvatore.

Rockwood agregó una y posteriormente dos más.

TIPO 4. Lesión igual a la del tipo 3, pero la clavícula se introduce dentro del trapecio.

TIPO 5. Se desgarran los ligamentos acromioclaviculares, coracoclaviculares, cápsulas además los músculos deltoides y trapecio separándose del extremo de la clavícula y haciendo que la articulación se separe tres veces más de las anteriores. Esta separación tan grande es apreciada como un gran defecto y la palpación tratando de aproximarla al acromion es conocida como "SIGNO DE LA TECLA".

TIPO 6. Muy rara; se debe a una abducción forzada y la clavícula se coloca detrás de los músculos del hueso coracoides y delante del mismo en su base.

Se suele producir por un golpe en el hombro y sobre el acromión; su importancia depende del compromiso de una serie de estructuras: el ligamento acromioclavicular, el ligamento coracoclavicular, cápsula articular, músculos deltoides y trapecio. Se manifiesta por dolor en la articulación acromioclavicular, deformación del hombro según el grado de afección.

Posteriormente y por persistencia del dolor en hombro derecho - se refería en algún informe clínico que ya lo presentaba el día del accidente - y fiebre, tras acudir a Urgencias, y encontrando en pruebas complementarias - analítica sanguínea con leucocitosis, hiperbilirrubinemia - y en pruebas de imagen una colección - y tras punción y estudio del pus extraído , es diagnosticada de 
absceso en zona infraclavicular derecha, que se extiende hasta zona axilar, y que tras estudio microbiológico se confirma debido a Streptococus Pyogenes.

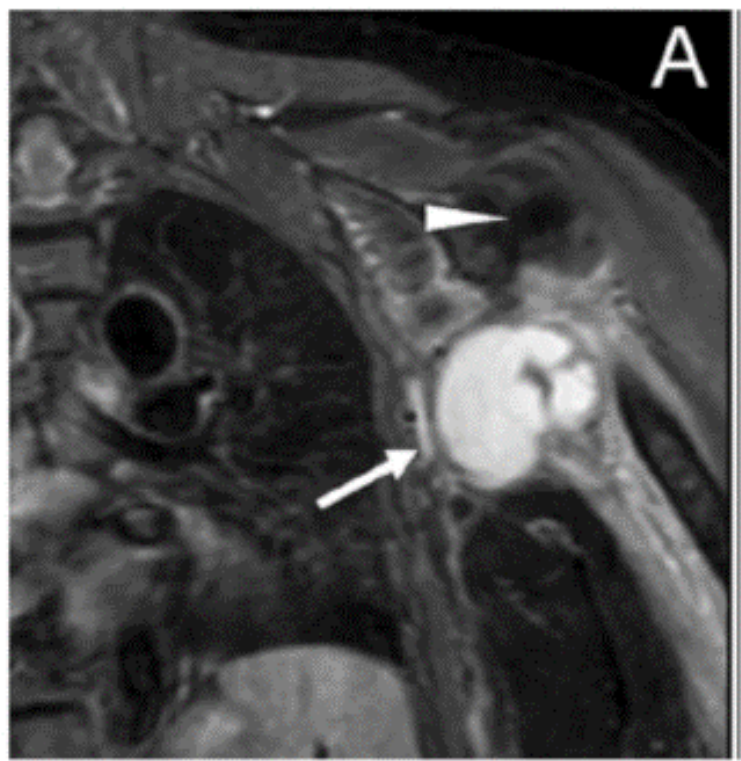

NOTA: NO corresponde a la paciente que comentamos

Fig 3. Absceso axilar por Streptoccocus Pyogenes

Se han descrito abscesos axilares complicados por trombosis venosa, debidas a infección por estreptococcus pyogenes (Kahn F. y cols., Axillary Abscess Complicated by Venous Thrombosis: Identification of Streptococcus Pyogenes by 16S PCR, J. Clinical Microbiology, Sep. 2010. 3435-3437), refiriéndose que el estreptococo piógeno o estreptococo grupo A es un patógeno humano importante, que produce una variedad de enfermedades que varían desde infecciones cutáneas leves como el impétigo, hasta fasciitis necrotizantes, con riesgo vital y síndrome tóxico similar a shock.

Se ha referido la formación de abscesos en tejido muscular (Abuelreish M. A., Rathore M.H., 2005. Subpectoral abscess a rare group A beta-hemolytic Streptococcus infection. Pediatr. Infect. Dis., J., 24:1121-1122, Bertelsen J., Severinsen M.T., Deep venous thrombosis caused by severe infection with group A streptococci, Ugeskr. Laeger, 168: 2260-2261, Vianii R.M., Bromberg K., Bradley J.S., Obturator internus muscle abscess in children; report of seven cases and review. Clin. Infect. Dis. 28:117-122). 
- En esta lesionada, se refería hinchazón en miembro superior derecho, por lo que quizá habría que haber realizado estudio para detectar complicación trombótica, caso de que persistieran signos en el momento actual.

McKay-Davies I. Tweedie D y cols., Unusual presentation of more common disease/injury, The perils of Paint-balling, BMJ Case Reports 2011, Doi: 10.1136, refieren absceso tras hematoma secundario a hiperextensión del cuello.

Así se han referido muchos casos de hematoma cervical tras hiperextensión brusca y violenta de cuello tras situaciones como ocurren con el latigazo cervical, y esos hematomas tienden a ocurrir en el espacio retrofaríngeo y menos en el parafaríngeo.

Los abscesos en hematoma tras hiperextensión de cuello son muy raros y sus signos incluyen fiebre, marcadores inflamatorios elevados, masa fluctuante con signos de inflamación local, asimetría orofaríngea o desplazamiento de las amígdalas, con signos posibles de complicaciones que incluyen trombosis venosa, tromboflebitis, mediastinitis, síndrome de Horner y sepsis.

También se han diagnosticado artritis sépticas acromioclaviculares con piomiositis contigua (Corey SA, Agger WA y cols, Acromioclavicular Septic Arthritis and Sternoclavicular Septic Artyhritis with Contiguous Pyomyositis, Clinics in Orthopedic Surgery 2015, 7;131-134).

Esta complicación es rara habiéndose descrito una incidencia de piomiositis de 0,5 por 100.000 personas/año, con sólo el 8\% de éstos casos ocurriendo en el hombro, siendo incluso más infrecuente en el músculo esternocleidomastoideo.

Se han descrito factores de riesgo para la piomiositis y la artritis séptica, tales como síndrome de inmuno-deficiencia adquirida (SIDA), diabetes, abuso de drogas intravenosas y cáncer.

Chiang AS, Ropiak ChR y cols, "Septic Arthritis of the Acromioclavicular Joint, A report of Four Cases", Bulletin of the NYU Hospital for Joint Diseases, 2007; 65(4):308-11, refieren que la artritis séptica de la articulación acromioclavicular es un diagnóstico infrecuente viéndose principalmente en paciente inmunocomprometidos.

La artritis séptica generalmente afecta a las articulaciones que soportan peso en la extremidad inferior comprendiendo del 61 al 79\% de los casos referidos de artritis séptica siendo la rodilla la articulación más afectada.

Mangas-Loria CAJ, Fuentes-Nucamendi MA y cols, "Septic arthritis of the acromioclavicular joint due to Streptococcus agalactiae. Case report", Rev. Med. Hosp. Gen. Mex. 2016, refieren otro caso de artritis séptica de la articulación acromioclavicular. 
Refieren que la incidencia de artritis séptica de la articulación acromioclavicular es del 2-10 por 100.000 en la población general aumentando en pacientes con prótesis articular; es más prevalente en varones y la edad media es de 54 años.

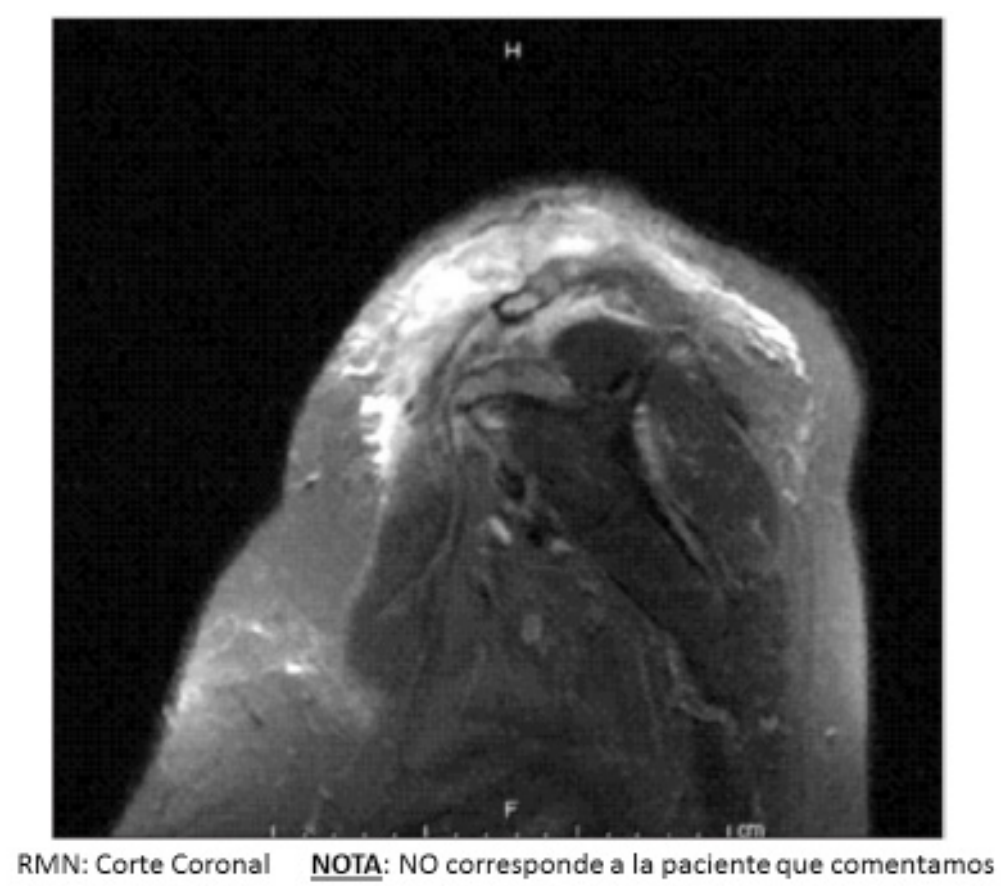

Fig 4. Artritis acromioclavicular septica y piomiositis de deltoides y supraespinoso

La artritis piogénica de la articulación acromioclavicular es una entidad rara (Martínez-Morillo M, Mateo Soria L, Riveros Frutos A, Tejera Segura B, Holgado Pérez S, Olivé Marqués A, Septicarthritis of the acromioclavicular joint: an uncommon location, Reumatol Clin. 2014 Jan-Feb;10(1):37-42). Los gérmenes responsables de la artritis séptica en la articulación acromiclavicular frecuentemente son del grupo de los estafilococos, aunque pueden ser causados por otras bacterias.

Otros autores también han descrito abscesos en músculos de la cintura escapular, (Sokolowski MJ, Koh JL, Pyomyositis of the shoulder girdle, Orthopedics. 2006 Nov;29 (11):1030-2).

Los abscesos por estreptococo se han descrito también en otras zonas corporales, como en zona subpectoral, pudiendo presentarse agudamente 
y con un curso `progresivo rápido, debiendo ser conscientes de sus presentaciones en zonas infrecuentes, (Abuelreish MA, Rathore MH, Subpectoral abscess a raregroup A beta-hemolytic Streptococcus infection, Pediatr Infect Dis J. 2005 Dec;24(12):1121-2) .

En el caso de la lesionada que comentamos, y respecto a los criterios de nexo causal para establecer una relación entre el accidente sufrido por esta conductora, consistente en una colisión por alcance posterior "a baja velocidad", se refieren lesiones iniciales consistentes en "esguince cervical", refiriéndose también en la información médica aportada, aunque no es totalmente coincidente, que la paciente tenía dolor en hombro derecho tras el accidente.

Transcurridos unos días, es diagnosticada de luxación acromioclavicular en hombro derecho, y posteriormente, por persistir molestias progresivas en hombro derecho y aparecer signos de infección (fiebre, enrojecimiento local, leucocitosis, hiperbilirrubinemia), se realizan pruebas de imagen - TC de hombro y tórax - que muestran absceso en región infraclavicular derecha hasta línea axilar media, cultivándose Estreptococo Pyogenes como gérmen causal.

Este absceso se habría formado sobre un pequeño hematoma local secundario a la luxación acromioclavicular, que siendo una complicación muy infrecuente, en ausencia de otra causa, se debe considerar debido al traumatismo secundario a una tracción sobre la articulación acromioclavicular, al llevar la mano sobre el volante y sufrir la colisión por alcance posterior. Así, respecto al criterio de localización, es compatible la lesión con el mecanismo lesivo.

Se han descrito lesiones en la articulación acromioclavicular por mecanismo de aceleración-desaceleración, aunque en los casos referidos, en general la lesión asentaba en el hombro por donde pasaba la banda torácica (oblícua) del cinturón de seguridad, Athoun E y cols, Lesión de la articulación acromioclavicular por aceleración-desaceleración como causa de dolor persistente en hombro: Pronóstico tras resección artroscópica, Indian J Orthop. 2014 Mar-Apr; 48 (2): 193-196., refiriéndose períodos de tiempo variables entre la fecha del accidente y la presentación del paciente con sintomas de afectación de la articulación acromioclavicular u otras zonas del hombro - Criterio cronológico.

En relación a otras causas posibles de la aparición del absceso referido, no se han objetivado otros posibles orígenes (Criterio de exclusión), y aunque en teoría podrían existir otras posibles causas (Ausencia de Criterio de Especificidad), no se han determinado en esta paciente. Atoun, Ehud et al. "Acromioclavicular Joint Acceleration-Deceleration Injury as a Cause of 
Persistent Shoulder Pain: Outcome after Arthroscopic Resection." Indian Journal of Orthopaedics 48.2 (2014): 193-196. PMC. Web. 23 June 2017.

El accidente consistió en una colisión por alcance posterior, en la que la lesionada conducía el turismo que fue golpeado en su zona posterior, sufriendo daños y deformaciones como consecuencia del impacto; se ha referido que en este tipo de colisiones, aunque no necesariamente tienen que existir daños visibles en los turismos implicados, para que se produzcan lesiones en sus ocupantes, en este caso, y aunque el accidente se puede considerar de los denominados de baja intensidad, el turismo conducido por la lesionada sufrió deformaciones, existiendo el criterio de intensidad, para explicar las lesiones sufridas por esta conductora.

EN CONCLUSION, aunque infrecuentes, pueden producirse evoluciones con complicaciones tras lesiones aparentemente banales en accidentes de tráfico con colisiones a baja velocidad, como es el caso que describimos, en el que las lesiones - iniciales, consistentes en "esguince cervical" - producido por un mecanismo de aceleración-desaceleración de la cabeza con transmisión de fuerza al cuello, y las diagnosticadas posteriormente - luxación acromioclavicular en hombro derecho - y mas tarde absceso en región infraclavicular derecha hasta línea axilar media, cultivándose Estreptococo Pyogenes como gérmen causal, (este absceso se habría formado sobre un pequeño hematoma local secundario a la luxación acromioclavicular), aunque pueden constituir una complicación muy infrecuente, en ausencia de otra causa, se deben considerar una complicación de lesión debida al traumatismo sobre el hombro, lesión secundaria a una tracción sobre la articulación acromioclavicular, al llevar la mano sobre el volante y sufrir la colisión por alcance posterior.

Agradecimientos: A Dñ ${ }^{a}$ Mónica Silva D’Arquea por su trabajo de mecanografiado 